\title{
RAGE-aptamer Attenuates the Growth and Liver Metastasis of Malignant Melanoma in Nude Mice
}

\author{
Nobutaka Nakamara, ${ }^{1}$ Takanori Matsui, ${ }^{1}$ Yuji Ishibashi, ${ }^{1}$ Ami Sotokawauchi, ${ }^{1}$ Kei Fukami, ${ }^{2}$ \\ Yuichiro Higashimoto, ${ }^{3}$ and Sho-ichi Yamagishi ${ }^{1}$
}

${ }^{1}$ Department of Pathophysiology and Therapeutics of Diabetic Vascular Complications; ${ }^{2}$ Department of Medicine; and ${ }^{3}$ Department of Chemistry, Kurume University School of Medicine, Kurume, Japan

\begin{abstract}
Epidemiological studies have suggested a link between cumulative diabetic exposure and cancer. The interaction of advanced glycation end products (AGEs) with their receptor (RAGE) may contribute to the phenomenon. We examined the effects of DNA aptamer raised against RAGE (RAGE-aptamer) on growth and liver metastasis of G361 melanoma in nude mice. Malignant melanoma cells were intradermally injected into the upper flank region of nude mice, which received continuous administration of RAGE-aptamer ( $38.4 \mathrm{pmol} / \mathrm{day} / \mathrm{g}$ body weight) or vehicle intraperitoneally by an osmotic pump up to $42 \mathrm{~d}$. RAGE-aptamer significantly reduced levels of 8-hydroxy-2'-deoxy-guanosine, AGEs, RAGE, proliferating nuclear antigen, cyclin D1, vascular endothelial growth factor (VEGF), monocyte chemoattractant protein-1 (MCP-1), and CD31 and Mac-3, respective markers of endothelial cells and macrophages in tumors of nude mice, and suppressed proliferation and liver metastasis of malignant melanoma. Furthermore, RAGE-aptamer attenuated AGE-induced oxidative stress generation, proliferation, and VEGF and MCP-1 gene expression in both G361 melanoma cells and endothelial cells. The present findings suggest that RAGE-aptamer could attenuate melanoma growth and liver metastasis in nude mice by suppressing tumor angiogenesis and macrophage infiltration via inhibition of the AGE-RAGE system. RAGE-aptamer may be a novel therapeutic tool for the treatment of malignant melanoma.
\end{abstract}

Online address: http://www.molmed.org

doi: $10.2119 / \mathrm{molmed} .2017 .00099$

\section{INTRODUCTION}

Diabetes and cancer are common chronic disorders observed in our aging society, both of which have been an increasing global health burden $(1,2)$. Furthermore, there is an accumulating body of clinical evidence that diabetes is associated with increased incidence and mortality rates of various types of cancers (3-6). Meta-analyses have confirmed that several types of cancers, such as pancreas, liver, colorectal and endometrial cancers, occur more frequently in diabetic patients, and mortality rates of breast, endometrial, colon and rectal cancers have been shown to be significantly increased in diabetic patients $(3,4)$. A prospective study in Sweden revealed that the risk of malignant melanoma, pancreatic cancer and urinary tract cancers was positively correlated with elevated fasting glucose values, whose association was totally independent of age, body mass index and smoking (5). Moreover, in a study by the Emerging Risk Factors Collaboration, the hazard ratio of cancer death was increased in type 2 diabetic patients (6). The positive correlation between diabetes and cancer death remained significant after additional adjustments

Address correspondence to Sho-ichi Yamagishi, Department of Pathophysiology and Therapeutics of Diabetic Vascular Complications, Kurume University School of Medicine, 67 Asahi-machi, Kurume 830-001 1, Japan. Phone: + 81-942-31-7873; Fax: +81-942-31-7895; E-mail: shoichi@med.kurume-u.ac.jp.

Submitted June 8, 2017; Accepted for Publication September 13, 2017;

Published Online (www.molmed.org) November 6, 2017.

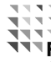

Feinstein Institute
for Medical Research

Northwell Health 
cumulative hyperglycemic exposure $(10,11)$. Furthermore, there is growing evidence that AGEs and their interaction with a receptor for AGEs (RAGE) stimulates proliferative and inflammatory reactions in various types of tissues, contributing to numerous diabetes- or aging-related disorders, including atherosclerotic cardiovascular disease, diabetic microangiopathy and tumor expansion and metastasis (12-16). Indeed, AGEs have been shown to cause proliferation or migration of several types of cancer cells, such as pancreatic cancer, breast cancer and malignant melanoma, via binding to RAGE (17-19). The findings suggest that suppression of AGE-RAGE in the tumor axis may be a novel therapeutic strategy for cancer growth and metastasis.

Aptamers are short, single-stranded oligonucleotides or peptides that are capable of binding to numerous types of target molecules through their versatile three-dimensional conformation with high affinity and specificity $(20,21)$. We have recently developed a DNA oligonucleotide aptamer directed against RAGE (RAGE-aptamer) and shown that it blocks the interaction of AGEs with RAGE and works as an antagonist for RAGE, thereby suppressing the AGEinduced inflammatory and fibrotic reactions in cultured mesangial cells (22). Furthermore, continuous intraperitoneal infusion of RAGE-aptamer has been found to reduce activation of the renal AGE-RAGE-oxidative stress system and attenuates podocyte damage, glomerular sclerosis and albuminuria, resulting in protection against the development and progression of streptozotocin-induced diabetic nephropathy in rats (22). Therefore, in the present study, we first examined the effects of RAGE-aptamer on the growth and liver metastasis of malignant melanoma in nude mice. To elucidate the molecular mechanism for the anti-tumor effects of RAGE-aptamer, we next studied in vitro whether RAGE-aptamer could inhibit proliferation and inflammatory reactions in AGE-exposed G361 melanoma cells and endothelial cells (ECs).

\section{MATERIALS AND METHODS}

\section{Materials}

Bovine serum albumin (BSA), Cohn fraction $\mathrm{V}, \mathrm{D}$-glyceraldehyde, $\mathrm{N}$ acetylcysteine (NAC), Dulbecco's modified Eagle's medium (DMEM) and cell proliferation reagent WST-1 were purchased from Sigma-Aldrich (St. Louis, MO, USA). G361 melanoma cells were obtained from American Type Culture Collection (Manassas, VA, USA). An osmotic minipump was purchased from Durect Co. (Cupertino, CA, USA). Monoclonal antibodies (Abs) raised against 8hydroxy-2'-deoxyguanosine (8-OHdG), an oxidative stress marker, were from Nikken SEIL Co., Ltd. (Shizuoka, Japan). Polyclonal Abs raised against RAGE, vascular endothelial growth factor (VEGF), CD31 and $\beta$-actin (Santa Cruz Biotechnology, Santa Cruz, CA, USA) were used in the present experiments. Mouse monoclonal and rabbit polyclonal Abs raised against cyclin D1 and p27, respectively, were purchased from Cell Signaling Technology (Beverly, MA, USA). Rabbit polyclonal Abs raised against proliferation cell nuclear antigen (PCNA), monocyte chemoattractant protein-1 (MCP-1) and CD107b (Mac-3) and rabbit monoclonal Abs against melanA were from Abcam (Cambridge, UK). Histofine Simple Stain MAX PO (MULTI) and M.O.M. immunodetection kit were purchased from Nichirei Co. (Tokyo, Japan) and Vector Lab (Burlingame, CA, USA), respectively. 5-(and-6)-carboxy-2', 7 '-dihydrofluorescein diacetate (carboxy- $\mathrm{H}_{2}$ DFFDA) was purchased from Thermo Fisher Scientific (San Jose, CA, USA).

\section{Preparation of AGEs}

AGE-modified BSA or nonglycated control BSA was prepared by incubating BSA $(25 \mathrm{mg} / \mathrm{mL})$ with or without $0.1 \mathrm{M}$ D-glyceraldehyde for $7 \mathrm{~d}$ (23).

\section{RAGE-aptamer Selection}

Using systemic evolution of ligands by exponential enrichment, RAGE-aptamer was prepared and selected (22). The sequence of phosphorothioate-modified RAGE-aptamer is ccTgATATggTgTcA ccgccgccTTAgTATTggTgTcTAc; phosphorothioate nucleotides are indicated as capital letters. We have already reported that RAGE-aptamer works as an antagonist for RAGE, and its dissociation constant $(\mathrm{Kd})$ is $5.68 \mathrm{nM}(22)$.

\section{Animal Experiments}

Six-week-old female athymic nude mice (Japan Clea, Tokyo) were used in the present experiments. Animal experiments were performed according to the following procedure (24). Two million G361 cells were intradermally administered into the upper flank of nude mice $(\mathrm{n}=14)$. RAGE-aptamer $(38.4 \mathrm{pmol} /$ day/g body weight, $\mathrm{n}=6$ ) or vehicle $(\mathrm{n}=8)$ was intraperitoneally administered to mice by an osmotic minipump. Tumor size and body weight were determined twice a week, and tumor volume $\left(\mathrm{mm}^{3}\right)$ was calculated by multiplying (largest diameter of tumor) $/ 2$ by (smallest diameter of tumor) ${ }^{2}$. At $42 \mathrm{~d}$ after tumor injection, mice were euthanized, and liver injury was evaluated by measuring aspartate aminotransferase (AST) and alanine aminotransferase (ALT) levels with a chemistry analyzer (FUJI DRI-CHEM 4000V; Fujifilm Co., Tokyo, Japan). G361 melanoma and liver were excised for immunohistochemical staining. All animal experiments were approved by the ethnical committee of Kurume University School of Medicine, Japan, and performed according to the Guide for the Care and Use of Laboratory Animals of the National Institutes of Health.

\section{Distribution and Kinetics of Continuously Infused RAGE-aptamer}

Eight-week-old female Balb/c-nu/ nu mice received continuous infusion (38.4 pmol/day/g body weight) of $\left[\gamma^{-32} \mathrm{P}\right]$ ATP-labeled RAGE-aptamer using an osmotic minipump, and were euthanized at $0,1,3$ and $7 \mathrm{~d}$ after the injection and at 3 and $7 \mathrm{~d}$ after stopping the injection (22). The half-life of RAGE-aptamer was estimated from the time required for the 
amount in skin after 7-d infusion to become reduced by half.

\section{Immunohistochemical Analyses}

Immunohistochemical staining was conducted as described previously $(24,25)$. G361 melanoma specimens were fixed, embedded and incubated overnight at $4^{\circ} \mathrm{C}$ with $\mathrm{Abs}$ raised against $8-\mathrm{OHdG}$, AGEs RAGE, PCNA, cyclin D1, p27, VEGF, CD31, MCP-1, CD107b (Mac-3) and melanA. Then the reactions were visualized with MAX PO (MULTI), except for cyclin D1 staining, whose expression was detected by M.O.M. immunodetection kit. Five different fields were obtained at random from each specimen, and immunoreactivity was measured by ImageJ.

\section{G361 Melanoma Cells and Human Umbilical Vein ECs}

G361 melanoma cells were maintained in DMEM containing 10\% fetal calf serum (24). In the experiments for cell proliferation, reverse transcription polymerase chain reaction (RT-PCR) and Western blot analyses, G361 cells were incubated with $1 \mathrm{mg} / \mathrm{mL}$ AGE-BSA or nonglycated control BSA with or without the indicated concentrations of RAGE-aptamer or NAC for 24 h. G361 cell experiments were performed in a medium supplemented with $1 \%$ fetal calf serum.

In the experiments of dihydroethidium (DHE) staining and RT-PCR analyses, human umbilical vein endothelial cells (HUVECs) (Lonza Group Ltd., Basel, Switzerland) were incubated with $50 \mu \mathrm{g} / \mathrm{mL}$ AGE-BSA or nonglycated control BSA with or without $100 \mathrm{nM}$ RAGE-aptamer for $4 \mathrm{~h}$ (26). HUVEC experiments were carried out in endothelial basal medium-2 containing $2 \%$ fetal calf serum, heparin and human fibroblast growth factor.

\section{Evaluation of Reactive Oxygen Species Generation in G361 Cells}

Intracellular reactive oxygen species (ROS) production in G361 cells was measured with a fluorescent probe, carboxy- $\mathrm{H}_{2}$ DFFDA (26). G361 cells were treated with or without $10 \mu \mathrm{M}$ carboxy- $\mathrm{H}_{2} \mathrm{DFFDA}$ for $1 \mathrm{~h}$. Then the cells were incubated with $1 \mathrm{mg} / \mathrm{mL}$ AGEBSA or nonglycated control BSA in the presence or absence of 1, 10 and $100 \mathrm{nM}$ RAGE-aptamer for $24 \mathrm{~min}$. Then intracellular superoxide production was measured by subtracting the fluorescence of cells treated without carboxy- $\mathrm{H}_{2}$ DFFDA from that with carboxy- $\mathrm{H}_{2}$ DFFDA.

\section{G361 Cell Proliferation Assay}

Proliferation of G361 cells was determined by WST-1-based colorimetric assay as described previously (24).

\section{ROS Generation of HUVECs}

HUVECs were incubated with $3 \mu \mathrm{M}$ DHE (Thermo Fisher Scientific, Waltham, MA, USA) for 30 min (27). ROS generation was evaluated by the fluorescence intensity obtained from five different fields of each sample using ImageJ.

\section{$\left({ }^{3} \mathrm{H}\right)$ Thymidine Incorporation into HUVECS}

HUVECs were incubated with $50 \mu \mathrm{g} / \mathrm{mL}$ AGE-BSA or nonglycated control BSA with or without $100 \mathrm{nM}$ RAGE-aptamer for $20 \mathrm{~h}$. After another $4 \mathrm{~h}$ incubation with $\left[{ }^{3} \mathrm{H}\right]$ thymidine, DNA synthesis was evaluated by measuring $\left[{ }^{3} \mathrm{H}\right]$ thymidine incorporation into HUVECs (28).

\section{Western Blot Analyses}

Western blots were conducted as described before (24). Membranes were probed with Abs raised against cyclin D1, p27 and $\beta$-actin. Bands were detected with an enhanced chemiluminescence detection system (GE Healthcare Japan).

\section{Real-Time RT-PCR Analyses}

Quantitative real-time RT-PCR was conducted using total RNA extracted from specimens of G361 melanoma, cultured G361 cells or HUVECs as described previously (26). Identification of primers for human G361 VEGF, HUVEC VEGF, MCP-1, vascular cell adhesion molecule-1 (VCAM-1), 18S rRNA and $\beta$-actin gene was Hs00900055_m1, Hs03929005_ m1, Hs00234140_m1, Hs00365486_m1,
Hs03003631_g1 and Hs01060665_g1 and Hs99999903_m1, respectively.

\section{Adhesion of THP- 1 Cells to HUVECs}

Adhesion of THP-1 cells to HUVECs was assayed as described previously (29). In brief, HUVECs were treated with $50 \mu \mathrm{g} / \mathrm{mL}$ AGE-BSA or nonglycated control BSA in the presence or absence of 100 nM RAGE-aptamer. After 24 h, HUVECs were incubated with BCECF-AM-labeled THP-1 cells for $4 \mathrm{~h}$, and then fluorescence intensities of the adherent THP-1 cells were measured.

\section{Tube Formation of HUVECs on Matrigel}

HUVECs were seeded on BD Biocoat Cellware Matrigel Basement Membrane Matrix 12-well plates (BD Bioscience, Franklin Lakes, NJ, USA) and then treated with $50 \mu \mathrm{g} / \mathrm{mL}$ nonglycated BSA or AGE-BSA in the presence or absence of $100 \mathrm{nM}$ RAGE-aptamer at $37^{\circ} \mathrm{C}$ for $2 \mathrm{~h}$. Four microscopic fields selected at random from each well $(\mathrm{N}=3)$ were photographed, and the length of tube-like structures was measured with microcomputer-assisted ImageJ (24).

\section{Statistical Analyses}

All values are shown as mean \pm standard deviation. Student $t$ test was performed for statistical comparison between the two animal groups. One-way analysis of variance followed by Tukey's test, Dunnett's test, Steel-Dwass test or Student $t$ test was performed for multiple comparisons; $p<0.05$ was considered statistically significant.

\section{RESULTS}

\section{Distribution and Kinetics of Infused RAGE-aptamer}

As shown in Figure 1A, continuously injected RAGE-aptamer for $7 \mathrm{~d}$ was distributed mainly in the adipose tissue, skin, muscle and kidney. A substantial amount of RAGE-aptamer remained in the skin and liver $7 \mathrm{~d}$ after stopping the infusion (Figure 1B). The half-life of infused RAGE-aptamer in the skin was $3.7 \pm 1.1 \mathrm{~d}$. 

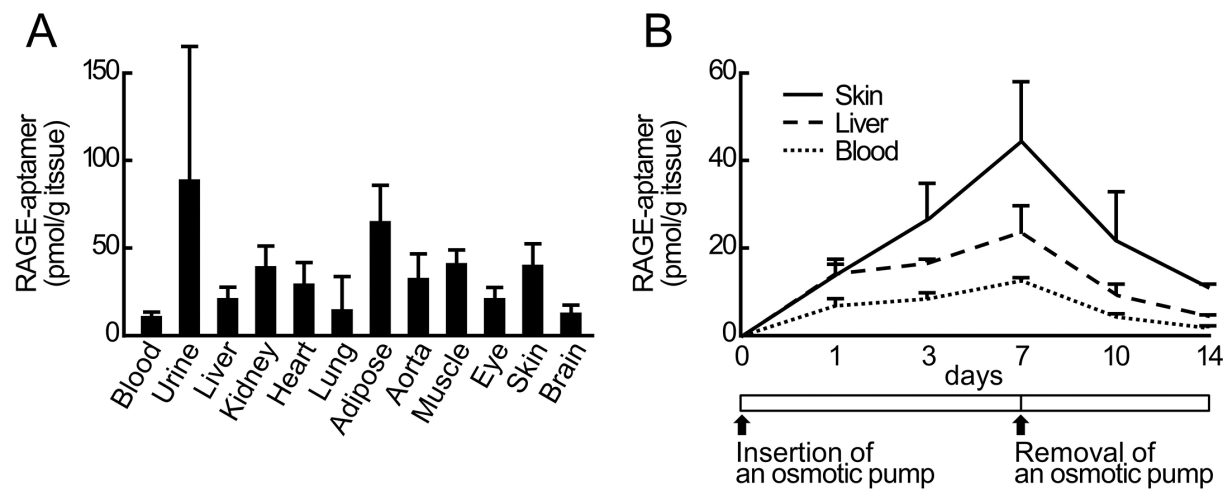

Figure 1. A, B. Biodistribution and time course kinetics of $\left(\gamma^{-32} \mathrm{P}\right)$ ATP-labeled RAGE-aptamer. Eight-week-old Balb/c-nu/nu mice received continuous intraperitoneal infusion of $\left(\gamma^{-}{ }^{32} \mathrm{P}\right)$ ATP-labeled RAGE-aptamer for $7 \mathrm{~d}$. Then blood, urine and several organs were obtained at the indicated time periods. $\left(\gamma^{3}{ }^{32}\right.$ P)ATP-labeled RAGE-aptamer was detected and measured by Cherenkov counting. Results are presented as pmol per gram of each tissue. $\mathrm{N}=4$ for each group.

\section{Effects of RAGE-aptamer on Tumor Expansion and Body Weight of Nude Mice}

We examined the effects of continuous injection of RAGE-aptamer on G361 tumor expansion of nude mice. As shown in Figures 2A and B, tumor size of G361 melanoma cells reached $400 \mathrm{~mm}^{3}$ in nude mice $42 \mathrm{~d}$ after intradermal tumor injection. Continuous intraperitoneal infusion of RAGE-aptamer for $28 \mathrm{~d}$ (from d 15 to d 42) significantly decreased the tumor volume, and tumor growth inhibitory effects of RAGE-aptamer remained significant up to $42 \mathrm{~d}$. At the end of the experiments, G361 tumor volume in RAGE-aptamer-treated mice was one-third of that in vehicle-treated mice (Figure 2B). From d 17 onward, the body weight of RAGE-aptamer-treated mice was significantly heavier than that of vehicle-treated mice (Figure 2C).

\section{Effects of RAGE-aptamer on Oxidative Stress, AGEs and RAGE in G361 Tumors of Nude Mice \\ We studied the effects of RAGE- aptamer on oxidative stress, AGE and RAGE levels in the G361 tumors of nude mice. As shown in Figures 3A-C, immu- nohistochemical analysis revealed that oxidative stress marker 8-OHdG, AGE and RAGE levels were significantly}

reduced in the G361 tumors of nude mice by the treatment of RAGE-aptamer.

\section{Effects of RAGE-aptamer on Tumor Proliferation, Tumor-Associated Angiogenesis and Macrophage Infiltration in Nude Mice}

We examined the effect of RAGEaptamer on tumor growth as well as tumor-associated angiogenesis and macrophage infiltration in nude mice. Immunohistochemical analysis showed that PCNA-positive cell levels were significantly lower in G361 melanoma of RAGE-aptamer-treated nude mice (Figure 4A). Furthermore, tumor gene and protein expression levels of cyclin D1, one of the G1 cyclins that promote passage through the G1 phase, and p27, a cyclin-dependent kinase inhibitor that causes G1 arrest, were decreased by the treatment of RAGE-aptamer (Figures 4B and C) (30). As shown in Figures 4D-G, RAGE-aptamer treatment for $28 \mathrm{~d}$ also significantly reduced tumor expression levels of VEGF, MCP-1, and CD31 and Mac-3, respective markers of ECs and macrophages in nude mice.

\section{Effects of RAGE-aptamer on Liver Metastasis of G361 Tumors in Nude Mice \\ We further examined whether RAGE- aptamer treatment could prevent liver}

metastasis of G361 tumors. RAGEaptamer treatment significantly prevented elevation of AST and ALT and attenuated liver metastasis of G361 tumors in nude mice (Figure 5). MelanA, a marker of melanoma, was not detected in normal non-tumor-bearing mice.

\section{Effects of RAGE-aptamer or NAC on ROS Generation, Proliferation, Cyclin D 1, p27, and VEGF and MCP- 1 Expression in G361 Melanoma Cells Exposed to AGEs}

As shown in Figure 6, AGEs significantly increased ROS generation, proliferation, cyclin D1 and p27 expression, and VEGF and MCP-1 mRNA levels in G361 melanoma cells, all of which were dose-dependently suppressed by RAGE-aptamer. NAC, an antioxidant, mimicked the beneficial effects of RAGE-aptamer on AGE-exposed G361 cells; 3 mM NAC significantly attenuated the deleterious effects of AGEs on G361 cells (Figure 6).

\section{Effects of RAGE-aptamer on ROS Generation, Proliferation and Inflammatory Reactions in, and Tube Formation of, AGE-exposed HUVECs}

As in the case in G361 cells, AGEs significantly stimulated superoxide generation, proliferation measured by $\left[{ }^{3} \mathrm{H}\right]$ thymidine incorporation, and VEGF, VCAM-1 and MCP-1 gene expression in, and THP-1 cell adhesion to, HUVECs, which were blocked by RAGE-aptamer (Figures 7A-F). Furthermore, RAGEaptamer significantly prevented an AGE-induced increase in tube formation of HUVECs on Matrigel (Figure 7G). One hundred $\mathrm{nM}$ of RAGE-aptamer alone did not affect ROS generation, VEGF, MCP-1 or VCAM-1 mRNA levels in nonglycated control BSA-exposed HUVECs (data not shown).

\section{DISCUSSION}

Accumulating evidence has shown active participation of the AGE-RAGE system in various tumor growth and metastasis pathways (16-19). Here we investigated the effects of RAGE-aptamer 
A
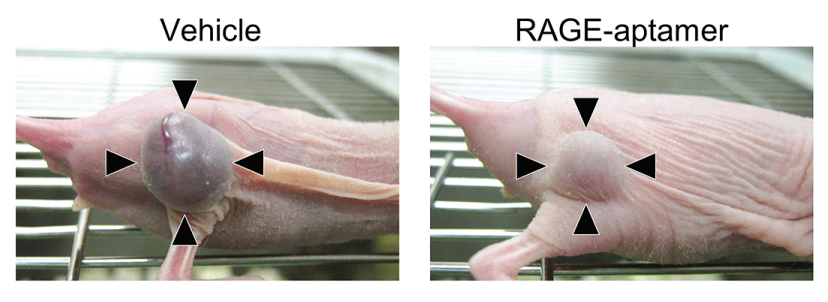

$\mathrm{B}$

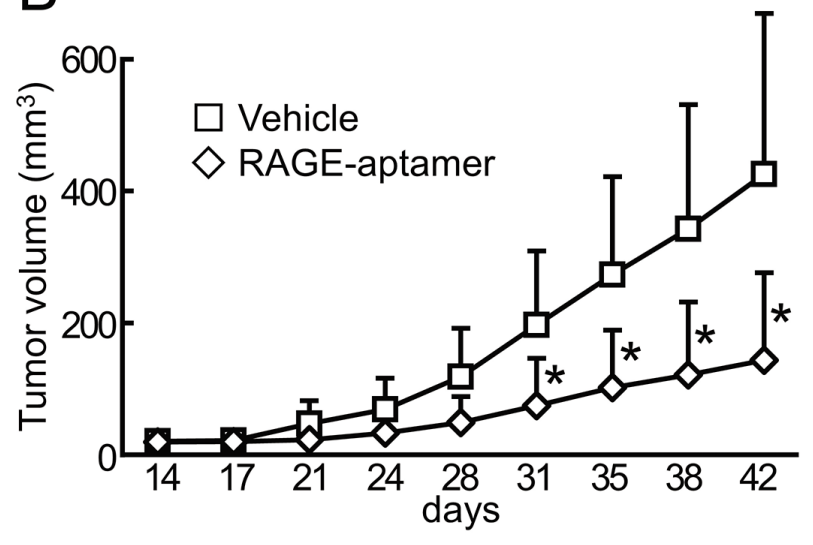

C

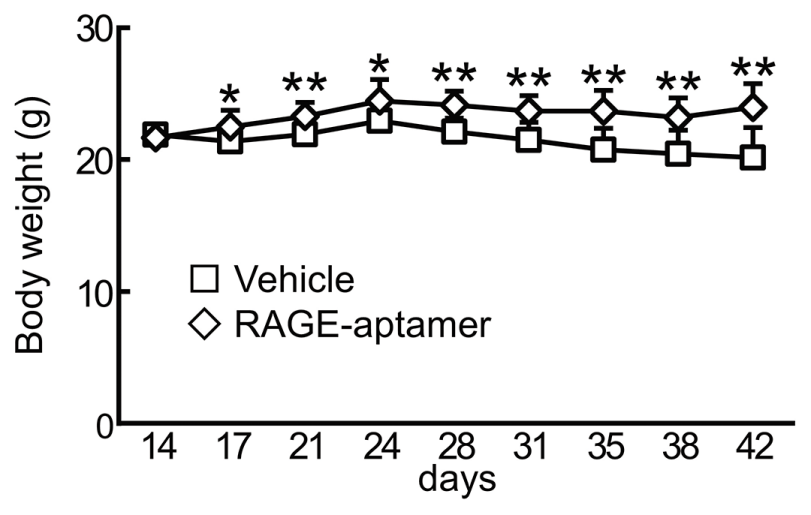

Figure 2. Effects of RAGE-aptamer on (A, B) tumor growth and (C) body weight in nude mice. Two million $G 361$ cells were intradermally administered into the upper flank of 6-wk-old female athymic nude mice $(\mathrm{N}=14)$. Mice received continuous intraperitoneal injection of RAGE-aptamer $(\mathrm{N}=6)$ or vehicle $(\mathrm{N}=8)$ by an osmotic minipump. Then tumor volume and body weight were measured twice a week. Panel A shows typical photographs of $\mathrm{G} 361$ tumors $42 \mathrm{~d}$ after each injection. Arrowheads indicate tumors. ${ }^{*}$ and ${ }^{* *}, p<0.05$ and $p<0.01$ compared with the values of vehicle-treated mice, respectively.

on melanoma growth and metastasis in nude mice. We found in this study that (1) continuous intraperitoneal administration of RAGE-aptamer for $28 \mathrm{~d}$ attenuated tumor growth and liver metastasis of G361 malignant melanoma in nude mice; (2) PCNA-positive proliferating cell levels were significantly reduced in RAGE-aptamer-treated G361 tumors in association with a decrease in cyclin D1, which is an allosteric stimulator of cell cycle transition from G1 to S phase and a marker for poor prognosis of patients with malignant melanoma (31,32); (3) expression levels of 8-OHdG, AGEs and RAGE in G361 melanoma were reduced by RAGE-aptamer; and (4) administration of RAGE-aptamer significantly decreased VEGF and MCP-1 levels in G361 tumors and subsequently suppressed tumor-associated angiogenesis and macrophage infiltration. The findings of the present study have extended our previous observations showing that (1) AGEs stimulated G361 melanoma cell proliferation, migration and invasion in vitro; (2) RAGE was overexpressed in human melanoma cells, including G361 cells; (3) anti-RAGE Abs inhibited AGE-induced proliferation of G361 melanoma cells; (4) DNA-aptamer raised against AGEs or RAGE-Abs suppressed the growth of implanted G361 melanoma in nude mice; and (5) Abs raised against RAGE inhibited the lung metastasis of G361 melanoma and resultantly prolonged the survival of tumor-bearing immunoincompetent mice $(18,24,33)$. Since RAGE-aptamer treatment for $28 \mathrm{~d}$ significantly ameliorated the elevation of liver transaminases and restored the decrease in body weight in nude mice, it is unlikely that RAGE-aptamer could exert nonspecific toxic effects in tumor-bearing mice. Furthermore, although RAGEaptamer decreased rather than increased expression of a cyclin-dependent kinase inhibitor, p27, in G361 tumors, it may be a reciprocal change, because PCNA, a nuclear antigen, which is expressed during the $S$ phase of the cell cycle, was reduced in RAGE-aptamer-treated tumors.

Although, as mentioned above, administration of neutralizing RAGE-Abs inhibited tumor growth and metastasis of G361 melanoma (18), aptamers have a number of potential advantages over Abs, which can increase their clinical applicability $(20,21)$. Indeed, aptamers do not stimulate inflammatory reactions and are thermally stable even after a $95^{\circ} \mathrm{C}$ denaturation, which characteristics sharply 

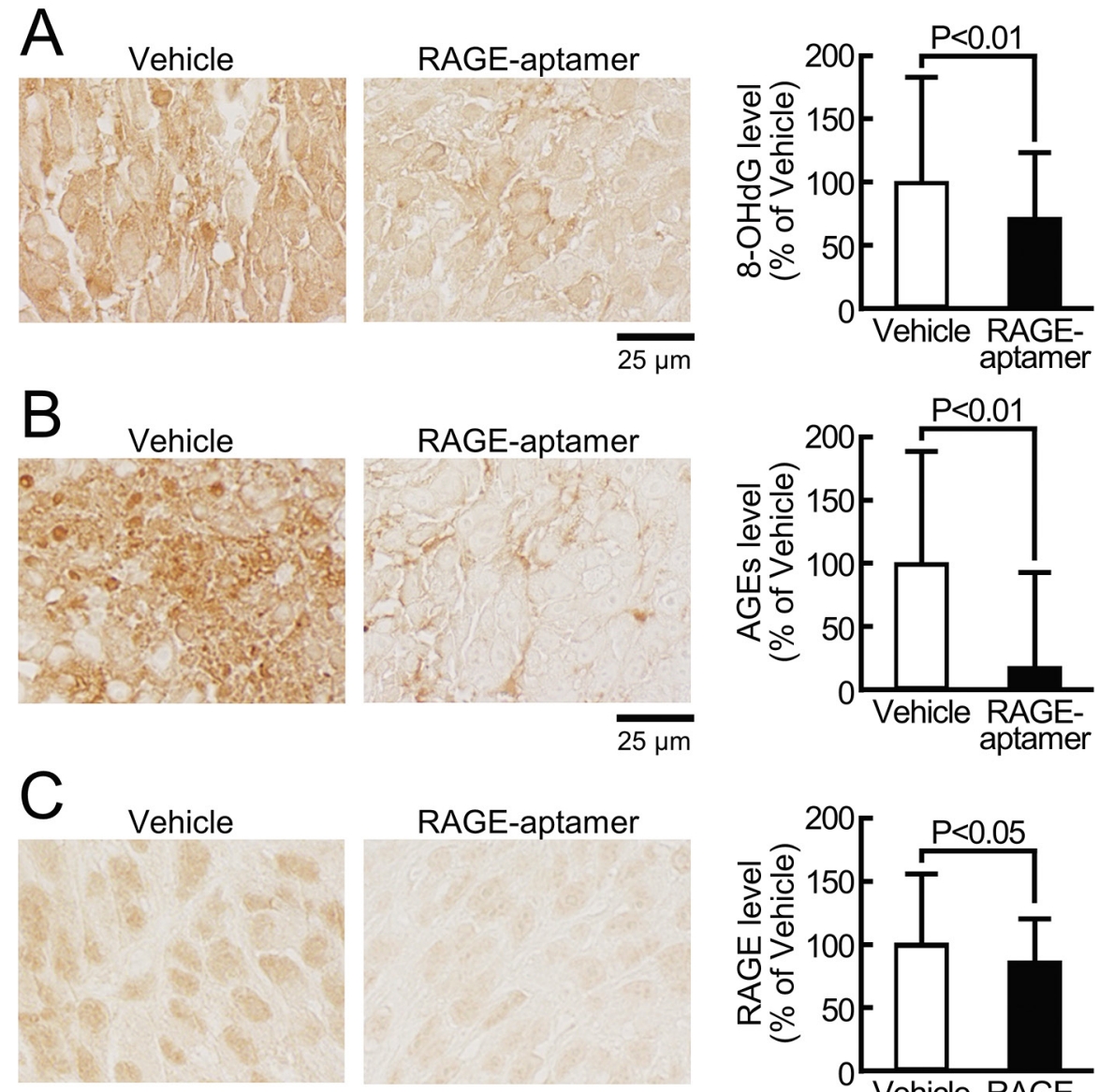

RAGE-aptamer

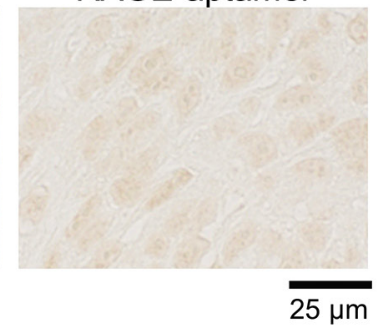

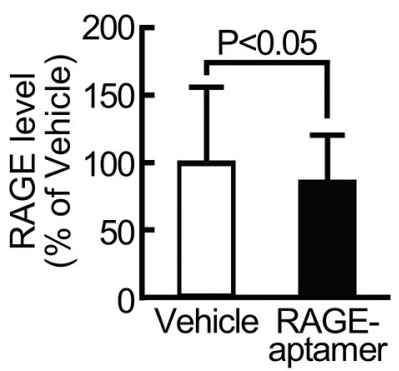

Figure 3. Effects of RAGE-aptamer on immunostaining of (A) 8-OHdG, (B) AGEs and (C) RAGE in G361 tumors. (A-C) Each left panel shows representative photographs of immunostaining. Quantitative data are shown in each right panel.

contrast with those of neutralizing Abs $(20,21)$. Moreover, aptamers can be massively synthesized in vitro with lower production cost and are able to target any protein or cell, whereas preparation of Abs is a time- and money-consuming process that requires an animal system; therefore, it is hard to make Abs raised against nonimmunogenic targets or toxins $(20,21)$. In addition, the pharmacokinetics of aptamers can be easily improved by chemical modification $(20,21)$. In the present study, RAGE-aptamer was still detected in the skin $7 \mathrm{~d}$ after stopping the injection, and its elimination half-life was $3.7 \mathrm{~d}$. Therefore, RAGE-aptamer may be a novel and promising therapeutic tool for attenuating the growth and metastasis of malignant melanoma.

To elucidate the molecular mechanism for growth inhibitory and antimetastatic effects of RAGE-aptamer, we performed in vitro experiments using cultured G361 cells and HUVECs. As a result, we obtained the following data: (1) RAGEaptamer dose-dependently blocked AGE-induced ROS generation, proliferation, cyclin D1 and p27 expression, and upregulation of VEGF and MCP-1 mRNA levels in G361 melanoma cells, whereas an antioxidant, NAC, mimicked all of the effects of RAGE-aptamer on G361 cells; and (2) RAGE-aptamer also suppressed ROS generation, proliferation, and upregulation of VEGF, VCAM-1 and MCP-1 mRNA levels in, adhesion of THP- 1 cells to, and tube formation of AGE-exposed HUVECs.

VEGF and MCP-1 have been shown to contribute to tumor angiogenesis and macrophage infiltration, and are considered key molecules that could promote melanoma proliferation and metastasis (34-40). Indeed, VEGF levels in melanoma are correlated with tumor-associated angiogenesis and macrophage infiltration, the extent of which is one of the clinical determinants of melanoma prognosis (34-37). Furthermore, mutant MCP-1 therapy reduced tumor-associated angiogenesis as well as proliferation of malignant melanoma in mice by inhibiting macrophage infiltration into tumors, another marker for tumor invasiveness, metastasis and poor prognosis in patients with melanoma (38-40). Therefore, the present study suggests that there are at least two molecular pathways by which RAGE-aptamer attenuates growth expansion and liver metastasis of malignant melanoma in nude mice: one is reduction of tumor-associated angiogenesis and macrophage infiltration through the suppression of AGE-induced VEGF and MCP-1 expression in both G361 cells and ECs, and the other is direct growth inhibition of melanoma cells by inducing G1 arrest via a decrease in cyclin D1 and PCNA. We have previously shown that the AGE-RAGE-driven ROS production not only stimulates angiogenesis through production of autocrine VEGF, but also elicits inflammatory reactions in ECs by MCP-1 induction $(29,41,42)$. Taken together, RAGE-aptamer might inhibit growth and metastasis of malignant melanoma partly by inhibiting the harmful effects of AGEs on both tumor cells and ECs, which could lead to the attenuation of tumor angiogenesis and macrophage infiltration partly via the reduction of VEGF and MCP-1 expression.

AGE formation is stimulated under hypoxia and/or inflammatory conditions $(25,43,44)$. So it is probable that the AGERAGE axis in G361 tumors of nude mice may be enhanced. Since engagement 

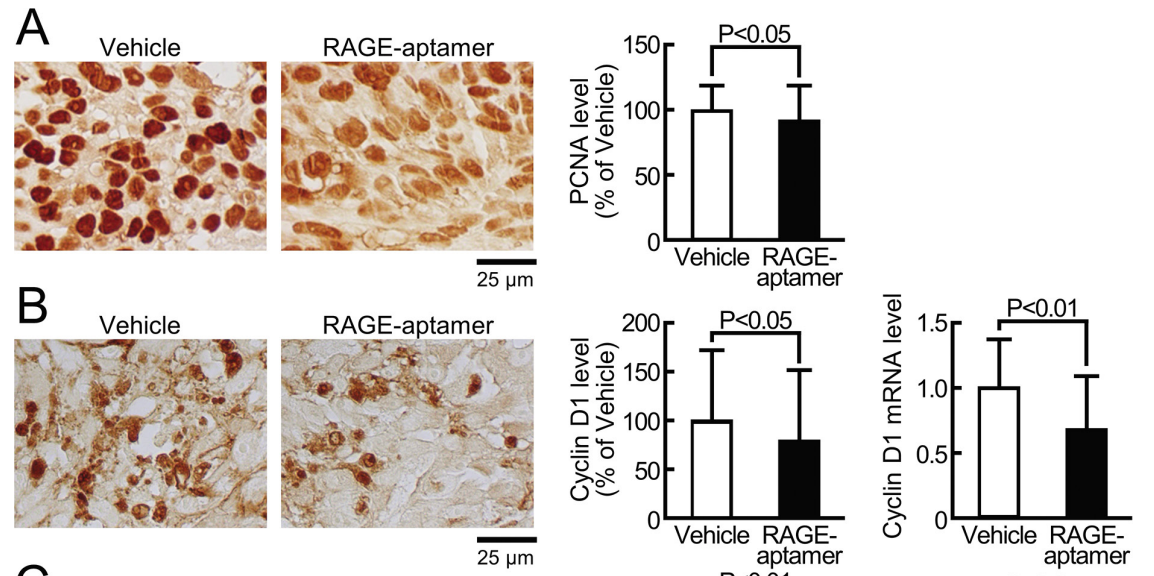
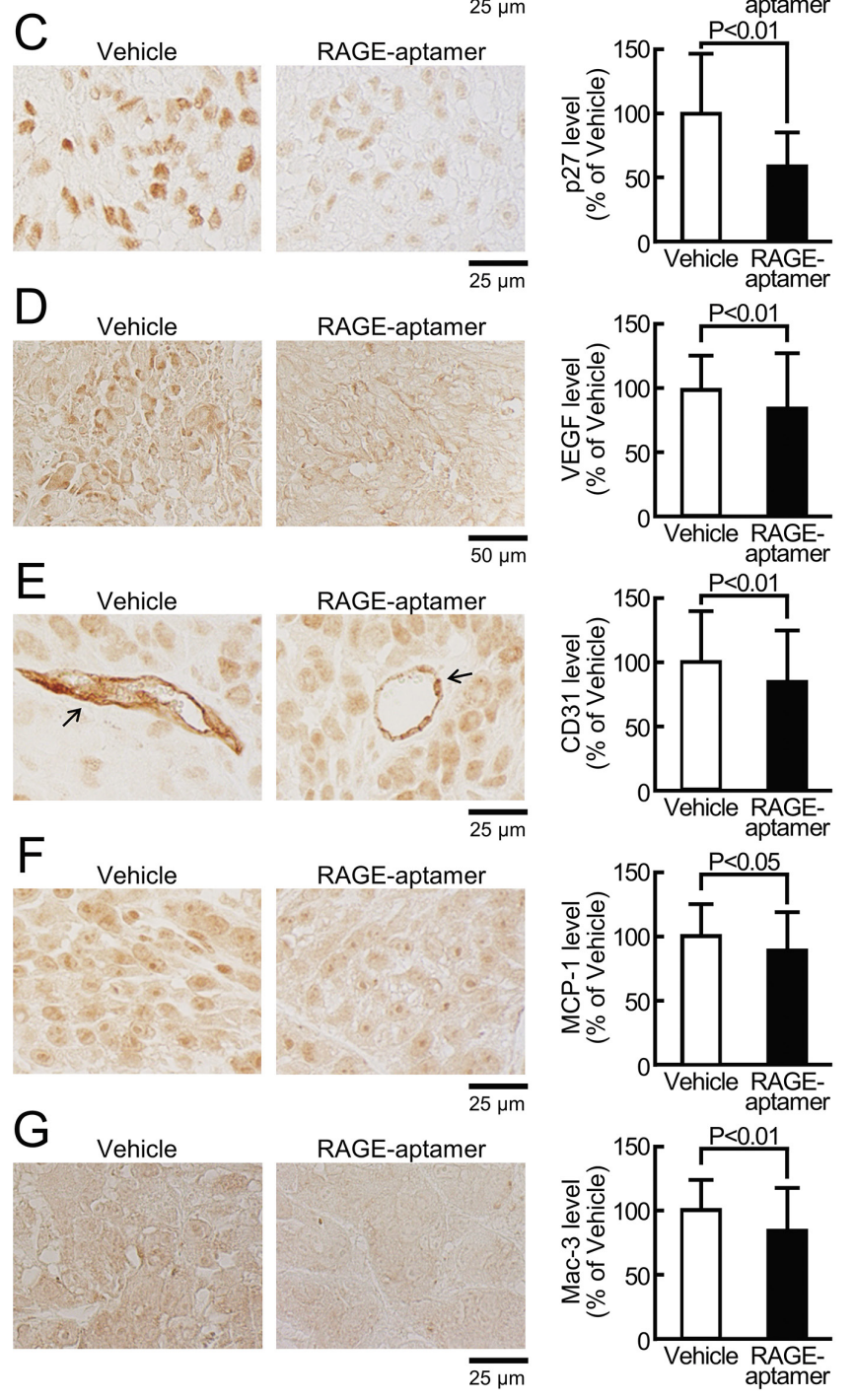
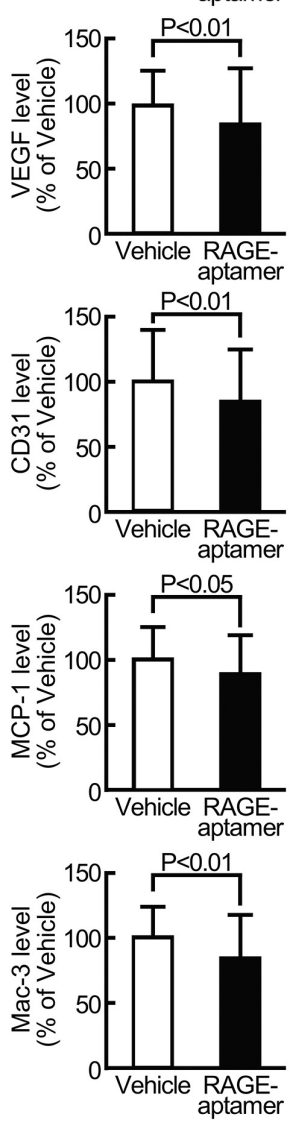
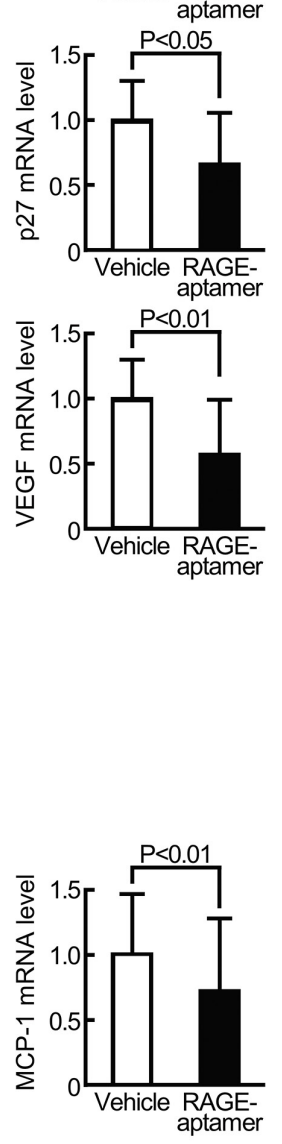

Figure 4. Effects of RAGE-aptamer on immunostaining of (A) PCNA, (B) cyclin DI, (C) p27, (D) VEGF, (E) CD31, (F) MCP-1 and (G) Mac-3 in G361 tumors. (A-G) Each left panel shows representative photographs of immunostaining. (E) Arrows indicate CD31-positive microvessels. Quantitative data are shown in each middle panel. (B) Cyclin D1, (C) p27, (D) VEGF and (F) MCP- 1 mRNA levels in G361 tumors are shown in the rightmost panel.
A

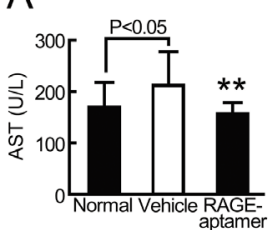

B

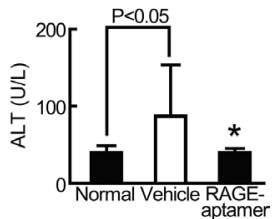

C
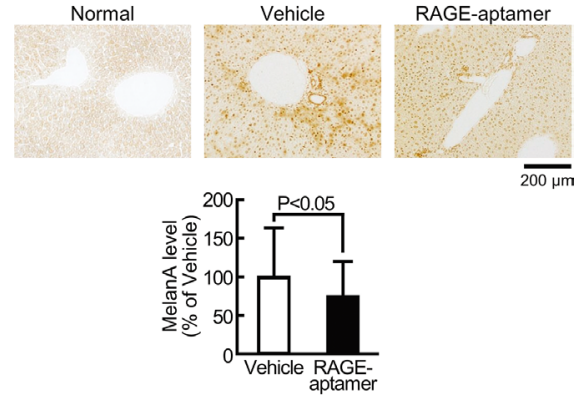

Figure 5. Effects of RAGE-aptamer on (A) AST, (B) ALT and (C) hepatic immunostaining of melanA in nude mice. (C) Upper panels show representative photographs of immunostaining. Lower panel shows quantitative data. ${ }^{*}$ and ${ }^{* *}, p<0.05$ and $p<0.01$ compared with the values of vehicle-treated mice, respectively.

of RAGE with AGEs in various tissues upregulates the expression of RAGE itself and further promotes the generation and accumulation of AGEs via ROS generation $(33,44,45)$, RAGE-aptamer may decrease the immunostaining levels of 8-OHdG, AGEs and RAGE in G361 tumors of nude mice by breaking the vicious cycle of the AGE-RAGE system in the tumor environment.

This study has several limitations. The primary drawback of this study is a lack of control experiments. However, $100 \mathrm{nM}$ RAGE-aptamer alone did not affect ROS generation, proliferation, cyclin D1 and p27 protein expression, or VEGF and MCP-1 mRNA levels in nonglycated control BSA-exposed G361 melanoma cells (Figure 6). Furthermore, since the halflife of RAGE-aptamer in the blood was about $2.9 \mathrm{~d}$ (Figure 1B), if we assume that the volume of distribution for RAGEaptamer is extracellular fluid and that total extracellular fluid volume is about $20 \%$ of body weight, the concentration 

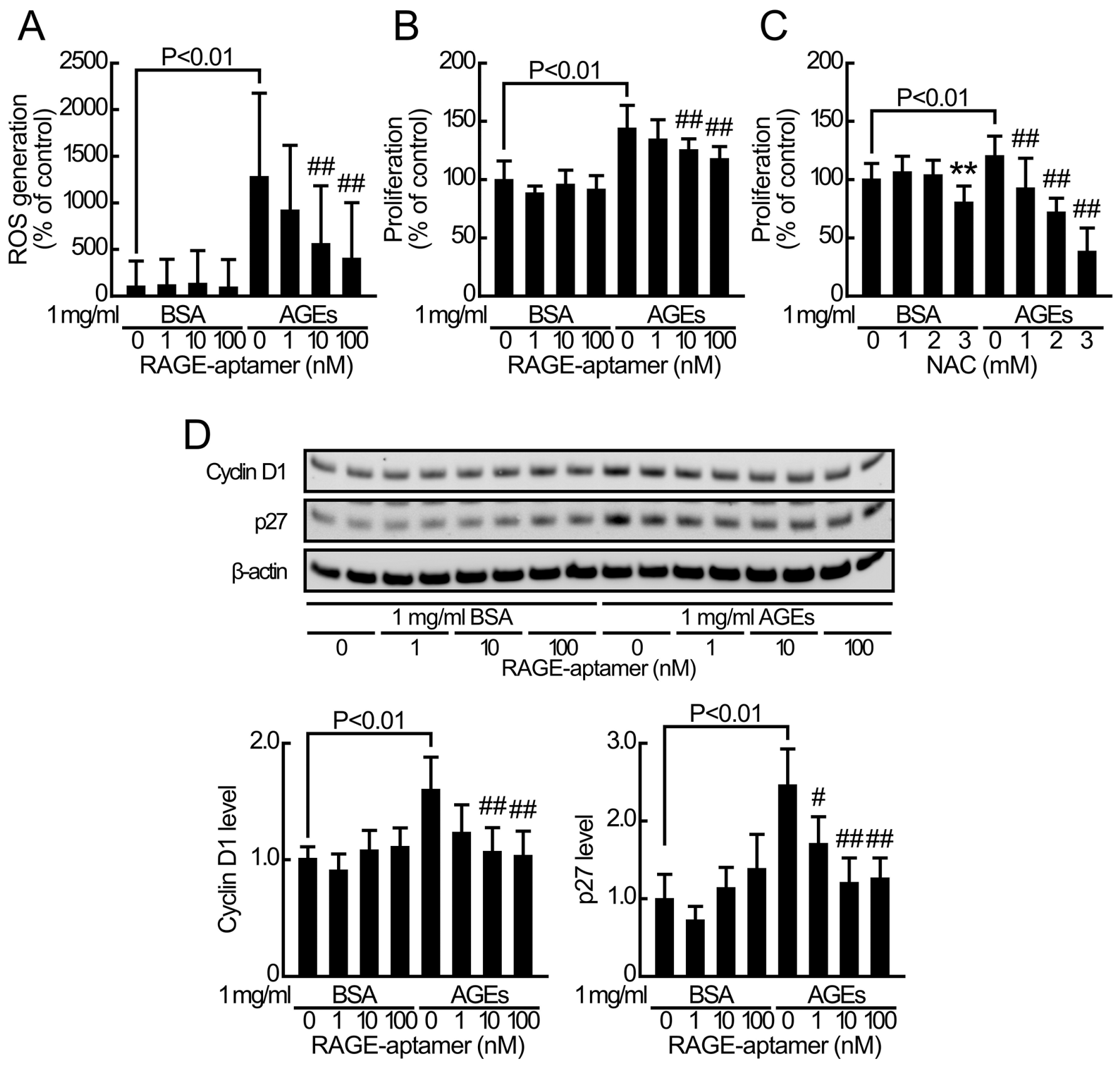

Figure 6. Effects of RAGE-aptamer or NAC on (A) ROS generation, (B, C) proliferation, (D, E) cyclin D1 and p27 protein levels, (F, G) VEGF and $(\mathrm{H}, \mathrm{I}) \mathrm{MCP}-1$ mRNA levels in AGE-exposed $G 361$ melanoma cells. (D, E) Each upper panel shows the bands of Western blot analysis for cyclin D1, p27 and $\beta$-actin, respectively. Quantitative data are shown in each lower panel. Data were normalized by the intensity of $\beta$-actin-derived signals and related to the value obtained with nonglycated BSA alone. (A) $N=6$ for each group. (B, $C, E$ ) $N=3$ for each group. (D) $N=5$ for each group. (F-I) $N=9$ for each group. ${ }^{* *}, P<0.01$ compared with the control value with nonglycated BSA alone. \# and \#\#, $p<0.05$ and $p<0.01$ compared with the value with AGEs alone, respectively.

Continued on the next page

of aptamer in extracellular fluid of tumor-bearing mice is estimated to be $150 \mathrm{nM}(38.4 / 0.2 \times 0.78 \mathrm{pmol} / \mathrm{mL})$, which is nearly equal to that used in vitro
(100 nM). Therefore, although in the present study we did not perform the control experiments using "scrambled" DNA aptamer of approximate molecular weight to the RAGE-aptamer, it is unlikely that RAGE-aptamer has nonspecific effects on the innate immune system, which could mediate its antitumor effects. 

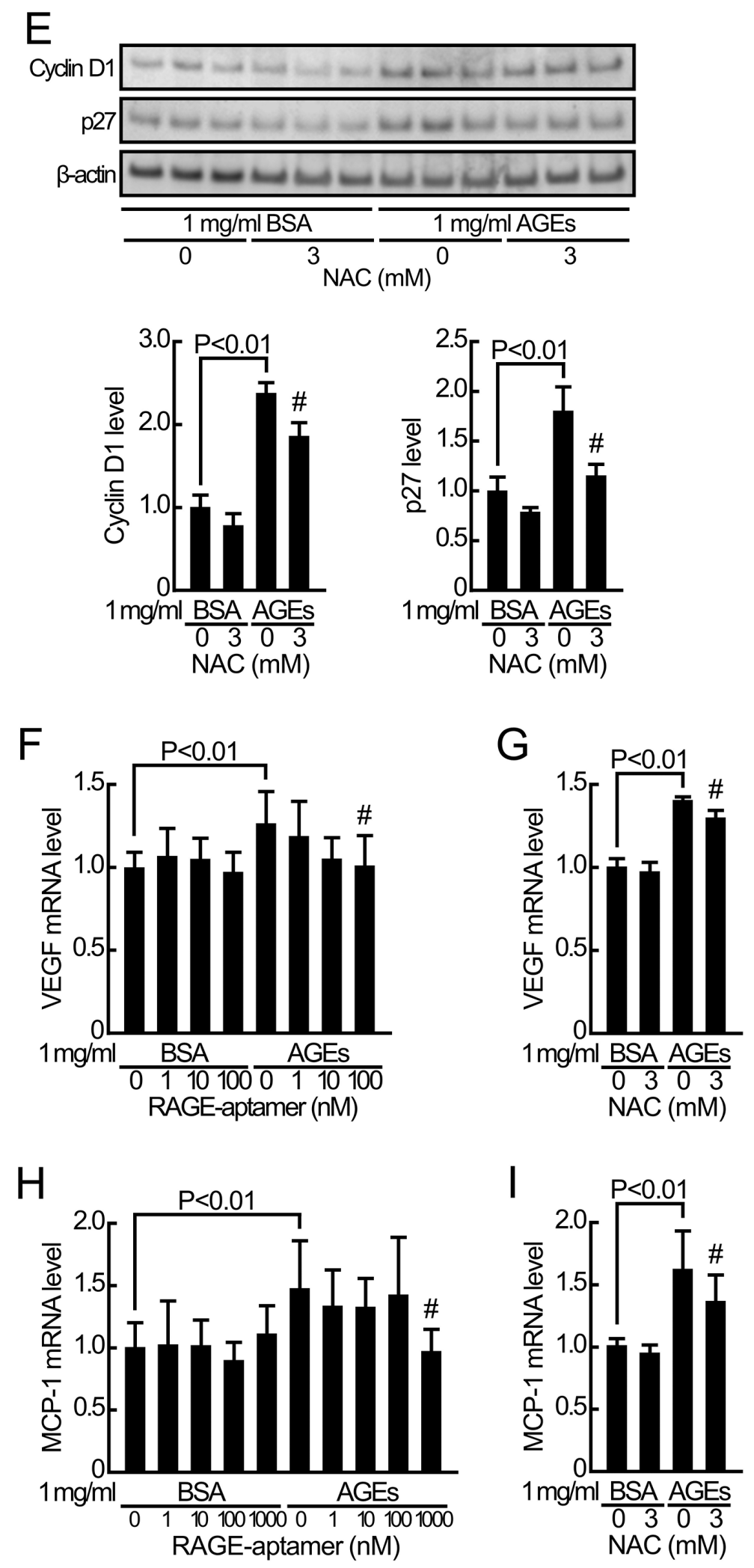

Figure 6. Continued.
As described above, although RAGE-aptamer treatment has some advantages over RAGE-Abs and the effects of RAGE-aptamer on G361 tumor growth were slightly stronger than those of RAGE-Abs (18), it would be helpful to see how its efficacy compares with other pharmacological inhibitors of RAGE, such as small interfering RNA directed against RAGE.

When the binding affinity of high mobility group box-1 (HMGB1), a more conventional RAGE ligand, to RAGE was evaluated in the presence or absence of RAGE-aptamer by quartz crystal microbalance (22), HMGB1 was found to bind to RAGE with a dissociation constant (Kd) of $48.3 \mathrm{nM}$, which value was significantly elevated to $8.97 \mu \mathrm{M}$. These observations suggest that RAGE-aptamer also inhibited the binding of HMGB1 to RAGE. Therefore, it would be interesting to further examine the efficacy of RAGE-aptamer in other diseases, such as sepsis, in which HMGB1 plays a pathological role (46).

\section{CONCLUSION}

In conclusion, the present findings suggest that RAGE-aptamer may attenuate melanoma growth and liver metastasis in nude mice by reducing tumor-associated angiogenesis and macrophage infiltration via suppression of the AGE-RAGE system. RAGE-aptamer may be a novel therapeutic tool for the treatment of melanoma.

\section{ACKNOWLEDGMENTS}

This work was supported in part by Grants-in-Aid for Scientific Research C (grant number 16K07101) (T.M.) from the Ministry of Education, Culture, Sports, Science, and Technology of Japan and by The Mitsubishi Foundation (ID 27147, S.Y.), Japan.

\section{DISCLOSURE}

The authors declare that they have no competing interests as defined by Molecular Medicine or other interests that might be perceived to influence the results and discussion reported in this paper. 

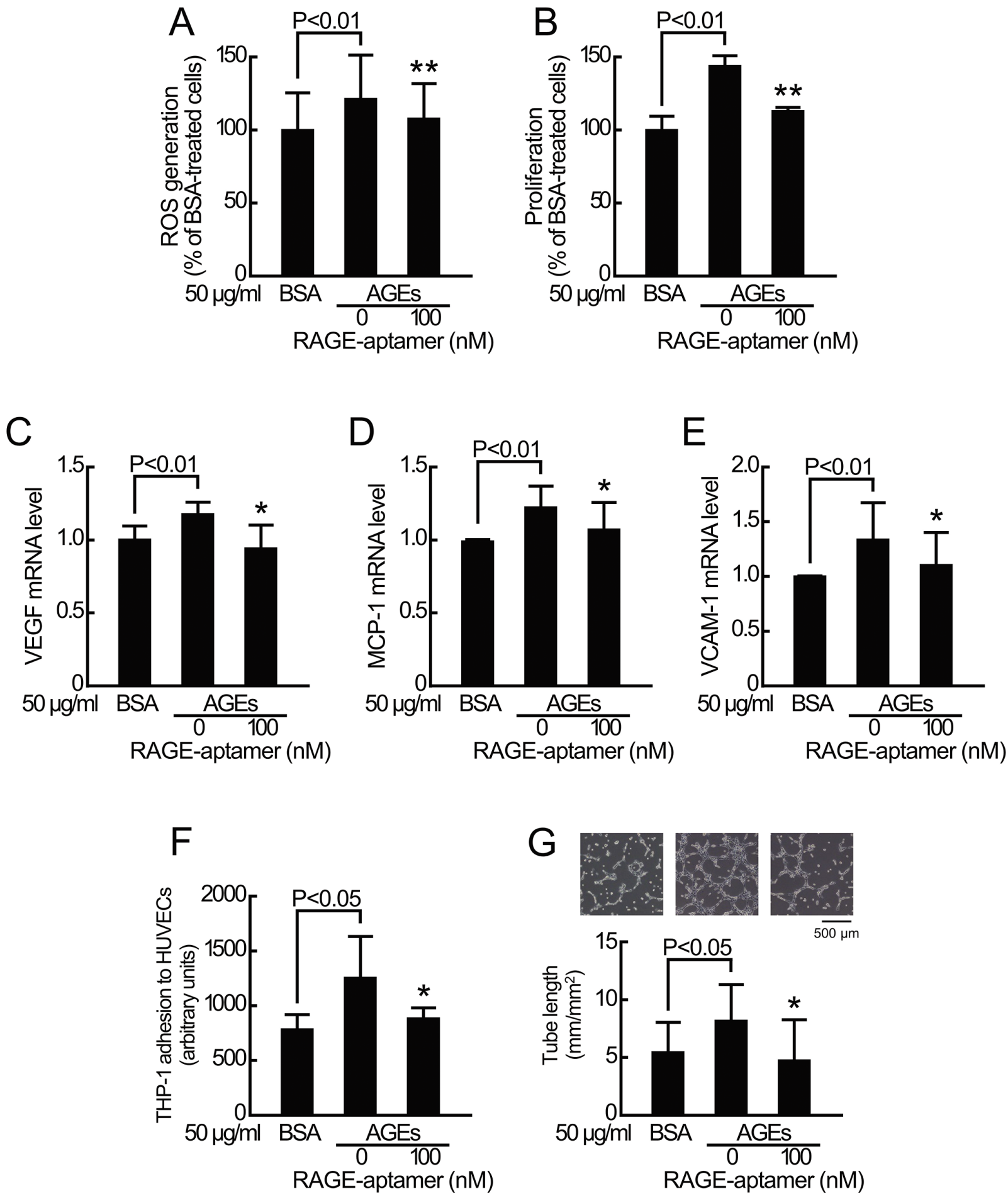

Figure 7. Effects of RAGE-aptamer on (A) ROS generation, (B) proliferation, (C) VEGF, (D) VCAM-1 and (E) MCP-1 mRNA levels in, (F) THP-1 cell adhesion to, and (G) tube formation of, AGE-exposed HUVECs. (A) Superoxide generation was evaluated by the fluorescence intensity of DHE staining. (B) Proliferation was determined by $\left({ }^{3} \mathrm{H}\right)$ thymidine incorporation into HUVECs. (C-E) Total RNA was transcribed and amplified by real-time PCR. Data were normalized by the intensity of $\beta$-actin-derived signals and related to the value obtained with nonglycated BSA alone. (G) Length of tube-like structures was measured. Upper panels show the representative microphotographs. (A) $N=3$ for each group. (B) $N=4$ for each group. (C) $N=6$ for each group. (D, $E) N=3$ for each group. (F) $N=6$ for each group. (G) $N=3$ for each group. ${ }^{*}$ and ${ }^{* *}, p<0.05$ and $p<0.01$ compared with the value with AGEs alone, respectively. 


\section{REFERENCES}

1. International Diabetes Federation. (2016) Diabetes Atlas, 7th ed. [Internet]. Available from www.diabetesatlas.org. Accessed May 21, 2017.

2. Braithwaite D, Boffetta P, Rebbeck TR, Meyskens F. (2012) Cancer prevention for global health: a report from the ASPO International Cancer Prevention Interest Group. Cancer Epidemiol. Biomarkers Prev. 21:1606-10.

3. Vigneri P, Frasca F, Sciacca L, Pandini G, Vigneri R. (2009) Diabetes and cancer. Endocr. Relat. Cancer. 16:1103-23.

4. Barone BB, et al. (2008) Long-term all-cause mortality in cancer patients with preexisting diabetes mellitus: a systematic review and meta-analysis. JAMA. 300:2754-64.

5. Stattin P, et al. (2007) Prospective study of hyperglycemia and cancer risk. Diabetes Care. 30:561-67.

6. Emerging Risk Factors Collaboration, Seshasai SR, et al. (2011) Diabetes mellitus, fasting glucose, and risk of cause-specific death. N. Engl. J. Med. 364:829-41.

7. Brownlee M, Cerami A, Vlassara H. (1988) Advanced glycosylation end products in tissue and the biochemical basis of diabetic complications. N. Engl. J. Med. 318:1315-21.

8. Dyer DG, Blackledge JA, Thorpe SR, Baynes JW (1991) Formation of pentosidine during nonenzymatic browning of proteins by glucose. Identification of glucose and other carbohydrates as possible precursors of pentosidine in vivo. J. Biol. Chem. 266:11654-60.

9. Genuth S, et al. (2005) Glycation and carboxymethyllysine levels in skin collagen predict the risk of future 10-year progression of diabetic retinopathy and nephropathy in the diabetes control and complications trial and epidemiology of diabetes interventions and complications participants with type 1 diabetes. Diabetes. 54:3103-11.

10. Bucala R. (2014) Diabetes, aging, and their tissue complications. J. Clin. Invest. 124:1887-88.

11. Yamagishi SI, Nakamura N, Matsui T. (2017) Glycation and cardiovascular disease in diabetes: A perspective on the concept of metabolic memory. J. Diabetes. 9:141-48.

12. Fleming TH, Humpert PM, Nawroth PP, Bierhaus A. (2011) Reactive metabolites and AGE/RAGE-mediated cellular dysfunction affect the aging process: a mini-review. Gerontology. 57:435-43.

13. Ramasamy R, Yan SF, Schmidt AM. (2011) Receptor for AGE (RAGE): signaling mechanisms in the pathogenesis of diabetes and its complications. Ann. NY Acad. Sci. 1243:88-102.

14. Ward MS, Fortheringham AK, Cooper ME, Forbes JM. (2013) Targeting advanced glycation endproducts and mitochondrial dysfunction in cardiovascular disease. Curr. Opin. Pharmacol. 13:654-61.

15. Chen M, Curtis TM, Stitt AW. (2013) Advanced glycation end products and diabetic retinopathy. Curr. Med. Chem. 20:3234-40.
16. Yamagishi S, Matsui T, Fukami K. (2015) Role of receptor for advanced glycation end products (RAGE) and its ligands in cancer risk. Rejuvenation Res. 18:48-56.

17. Yamamoto Y, Yamagishi S, Hsu CC, Yamamoto H. (1996) Advanced glycation end products: receptor interactions stimulate the growth of human pancreatic cancer cells through the induction of platelet-derived growth factor-B Biochem. Biophys. Res. Commun. 222:700-05.

18. Abe R, et al. (2004) Regulation of human melanoma growth and metastasis by AGE-AGE receptor interactions. J. Invest. Dermatol. 122:461-67.

19. Ishibashi $Y$, Matsui T, Takeuchi M, Yamagishi S. (2013) Metformin inhibits advanced glycation end products (AGEs)-induced growth and VEGF expression in MCF-7 breast cancer cells by suppressing AGEs receptor expression via AMP-activated protein kinase. Horm. Metab. Res. 45:387-390.

20. Ellington AD, Szostak JW. (1990) In vitro selection of RNA molecules that bind specific ligands. Nature. 346:818-22.

21. Sun H, Zu Y. (2015) A highlight of recent advances in aptamer technology and its application. Molecules. 20:11959-80.

22. Matsui T, et al. (2017) RAGE-aptamer blocks the development and progression of experimental diabetic nephropathy. Diabetes. 66:1683-95.

23. Yamagishi S, et al. (2006) Pigment epitheliumderived factor inhibits advanced glycation end product-induced retinal vascular hyperpermeability by blocking reactive oxygen speciesmediated vascular endothelial growth factor expression. J. Biol. Chem. 281:20213-20.

24. Ojima A, et al. (2014) DNA aptamer raised against advanced glycation end products inhibits melanoma growth in nude mice. Lab. Invest. 94:422-29.

25. Matsui T, Nishino Y, Takeuchi M, Yamagishi S. (2011) Vildagliptin blocks vascular injury in thoracic aorta of diabetic rats by suppressing advanced glycation end product-receptor axis. Pharmacol. Res. 63:383-88.

26. Matsui T, Oda E, Higashimoto Y, Yamagishi S. (2015) Glyceraldehyde-derived pyridinium (GLAP) evokes oxidative stress and inflammatory and thrombogenic reactions in endothelial cells via the interaction with RAGE. Cardiovasc. Diabetol. 14:1.

27. Ishibashi Y, Matsui T, Yamagishi S. (2016) Tofogliflozin, a highly selective inhibitor of SGLT2, blocks proinflammatory and proapoptotic effects of glucose overload on proximal tubular cells partly by suppressing oxidative stress generation. Horm. Metab. Res. 48:191-95.

28. Higashimoto $Y$, et al. (2007) In vitro selection of DNA aptamers that block toxic effects of AGE on cultured retinal pericytes. Microvasc. Res. 74:65-69.

29. Ishibashi Y, Matsui T, Ueda S, Fukami K, Yamagishi S. (2014) Advanced glycation end products potentiate citrated plasma-evoked oxidative and inflammatory reactions in endothelial cells by up-regulating proteaseactivated receptor-1 expression. Cardiovasc. Diabetol. 13:60.

30. Nakamura K, et al. (2007) Pigment epitheliumderived factor inhibits neointimal hyperplasia after vascular injury by blocking NADPH oxidase-mediated reactive oxygen species generation. Am. J. Pathol. 170:2159-70.

31. Cassarino DS, Cabral ES, Kartha RV, Swetter SM. (2008) Primary dermal melanoma: distinct immunohistochemical findings and clinical outcome compared with nodular and metastatic melanoma. Arch. Dermatol. 144:49-56.

32. Gammon B, Ali L, Guitart J, Gerami P. (2012) Homogeneous staining regions for cyclin D1, a marker of poor prognosis in malignant melanoma. Am. J. Dermatopathol. 34:487-90.

33. Abe R, Yamagishi S. (2008) AGE-RAGE system and carcinogenesis. Curr. Pharm. Des. 14:940-55.

34. Yamagishi S, et al. (2004) Minodronate, a newly developed nitrogen-containing bisphosphonate, suppresses melanoma growth and improves survival in nude mice by blocking vascular endothelial growth factor signaling. Am. J. Pathol. 165:1865-74.

35. Senger DR, et al. (1993) Vascular permeability factor in tumor biology. Cancer Metastasis Rev. 12:303-24.

36. Torisu H, et al. (2000) Macrophage infiltration correlates with tumor stage and angiogenesis in human malignant melanoma: possible involvement of TNFalpha and IL-1alpha. Int. J. Cancer. 85:182-88.

37. Salven P, Heikkila P, Joensuu H. (1997) Enhanced expression of vascular endothelial growth factor in metastatic melanoma. Br. J. Cancer. 76:930-34.

38. Koga M, et al. (2008) Mutant MCP-1 therapy inhibits tumor angiogenesis and growth of malignant melanoma in mice. Biochem. Biophys. Res. Commun. 365:279-84.

39. Shi L, et al. (2010) Clinicopathological implications of tumour-associated macrophages and vascularization in sinonasal melanoma. J. Int. Med. Res. 38:1276-86.

40. Chen P, et al. (2011) Tumor-associated macrophages promote angiogenesis and melanoma growth via adrenomedullin in a paracrine and autocrine manner. Clin. Cancer Res. 17:7230-39.

41. Yamagishi S, et al. (1997) Advanced glycation end products-driven angiogenesis in vitro. Induction of the growth and tube formation of human microvascular endothelial cells through autocrine vascular endothelial growth factor. J. Biol. Chem. 272:8723-30.

42. Matsui T, Nakamura N, Ojima A, Nishino Y, Yamagishi SI. (2016) Sulforaphane reduces advanced glycation end products (AGEs)-induced inflammation in endothelial cells and rat aorta. Nutr. Metab. Cardiovasc. Dis. 26:797-807.

43. Chang JS, et al. (2008) Oxygen deprivation triggers upregulation of early growth response-1 
RAGE-APTAMER AND MELANOMA

by the receptor for advanced glycation end products. Circ. Res. 102:905-13.

44. Kaida Y, et al. (2013) DNA aptamer raised against AGEs blocks the progression of experimental diabetic nephropathy. Diabetes. 62:3241-50.

45. Soro-Paavonen A, et al. (2008) Receptor for advanced glycation end products (RAGE) deficiency attenuates the development of atherosclerosis in diabetes. Diabetes. 57:2461-69.

46. Yang H, Wang H, Chavan SS, Andersson U. (2015) High Mobility Group Box Protein 1 (HMGB1): The Prototypical Endogenous Danger Molecule. Mol. Med. 21 (Suppl 1):S6-12.

Cite this article as: Nakamara N, et al. (2017) RAGE-aptamer attenuates the growth and liver metastasis of malignant melanoma in nude mice. Mol. Med. 23:295-306. 